\title{
Análisis vibratorio de placas armónicas en la guitarra acústica
}

\section{Vibration analysis of top plates of acoustic guitars}

\author{
Nicolás Acuña C. ${ }^{1}$, José Luis Barros ${ }^{2}$ \\ 1 Instituto de Acústica, Facultad de Ciencias de la Ingeniería Universidad Austral de Chile, \\ General Lagos 2086, Valdivia, nacuna86@gmail.com \\ 2 Instituto de Acústica, Facultad de Ciencias de la Ingeniería Universidad Austral de Chile, \\ General Lagos 2086, Valdivia, jbarros@uach.cl
}

\begin{abstract}
RESUMEN
El presente trabajo plantea la experimentación vibratoria con dos placas superiores de guitarras de distintas características en cuanto a calidad y procedencia de materiales y con un barraje tradicional del tipo Torres, en condiciones de borde libres, es decir, desacopladas del resto del cuerpo de la guitarra. Mediante el empleo del método tradicional de Chladni, se visualizan y localizan los modos de vibración más importantes. Por otra parte, se realiza la medición de la amplitud de vibración a través de la utilización de instrumentos adecuados, a fin de observar los efectos cuantitativos de la variabilidad de condiciones materiales y la influencia del barraje que posee cada placa armónica. El desarrollo de esta experiencia permite la identificación de los modos de vibración y patrones de sonido de radiación en condiciones libres. El método considera el análisis del comportamiento vibratorio de la placa de armónicos, permitiendo lograr un conocimiento general de su comportamiento con respecto a la adhesión de barras (en masa) y su disposición. No se consideran efectos posteriores como el acoplamiento a la guitarra y la incorporación del barniz.

Palabras clave: luthier, sistemas de barraje, placa armónica, cimática, método de Chladni, patrones de radiación sonora, análisis armónico.
\end{abstract}

\begin{abstract}
The present work raises from the vibratory experimentation with two top plates of guitars of different characteristics as for quality and origin of materials, with a traditional Torres bracing, at free edge conditions, disconnected of the guitar's body. By employing the Chladni's Traditional Method, the most important vibrational modes are visualized and located. Furthermore, the measurement of vibratory amplitude is analyzed through the utilization of suitable instruments, in order
\end{abstract}


to observe the quantitative effects of the material variability conditions and the bracing influence that every harmonic plate possesses. The development of this experience allows the identification of vibrational modes and sound radiation patterns in free vibrational conditions. The method consists on the solely isolation of the analysis for vibratory behavior to the harmonic plate. In this way, an overall knowledge of its behavior with respect to bars adhesion (mass) and its arrangement can be acquired. However, the subsequent effect of processes such as coupling or varnished, among others, are not considered.

Key Words: luthier, bars systems, armonic plate, cymatics, Chladni's method, sound radiation patterns, armonic analysis.

\section{INTRODUCCIÓN}

Aproximadamente desde finales del siglo XVIII y hasta nuestros días, la calibración sonora en placas de guitarra, así como de otros instrumentos cordófonos de madera (tales como el laúd o el violín), se ha llevado a cabo mediante la técnica del arreglo de barrajes en las distintas placas que forman el sistema (placas anteriores y posteriores), otorgando tensión en puntos estratégicos, para así manejar el "centro sonoro" del instrumento. Esto se complementa con la clásica técnica de "golpeteo" del luthier, que busca puntos de máxima vibración en la placa y con los ajustes posteriores del proceso constructivo de guitarras (acoplamiento al cuerpo, barnizado, entre otros).

De acuerdo a este procedimiento, la pieza que reviste mayor importancia es la placa superior o tapa armónica, pues es ésta la que tiene mayor influencia en la propagación del sonido hacia él auditor y el ejecutante, en todo el rango de frecuencias de la guitarra, y por lo tanto tiene directa relación con ciertos aspectos acústicos tales como los modos de radiación sonora y niveles de presión logrados, como también en factores musicales de extrema importancia tales como la afinación, timbre, sensibilidad a la dinámica, entre muchos otros. El barraje tradicional de la guitarra moderna del tipo Torres, se presenta como el punto de partida para un tipo de afinación estándar que se ha conservado hasta nuestros días. Sin embargo, diversas técnicas de ordenamiento de barras han sido desarrolladas y probadas a lo largo de la evolución paralela de la estructura de la guitarra y la música, que a través de sus requerimientos por parte de ejecutantes y también de compositores ha generado cambios constructivos en la estructura del sistema.

El siguiente trabajo analiza el comportamiento vibratorio en relación al barraje aplicado sobre una placa armónica en condiciones de borde libres, es decir sin acople al resto de la estructura del cuerpo de la guitarra, de una manera cuantitativa y visual en dos placas armónicas con distintas condiciones en cuanto a su procedencia y estado de construcción. Con este objeto, se realiza una identificación visual a través del tradicional método de Chladni y luego una medición de la respuesta en aceleración para cada caso, contraponiendo de esta forma los diversos factores constructivos 
de la manufactura detallada de un luthier versus la construcción en serie de guitarras, donde se tiene especial atención en la influencia del sistema de barraje.

\section{FUNDAMENTOS TEÓRICOS}

Es posible pensar en el comportamiento vibrante de una placa armónica como un pistón que se acopla como oscilador armónico simple al resto del cuerpo de la guitarra: cavidad de aire, flancos y placa posterior, planteamiento realizado por Christensen [2].

De acuerdo a este razonamiento, se tiene la ecuación de movimiento con un desplazamiento $x_{t}$ y $k_{t}$ como la constante de elasticidad de Hooke:

$$
\frac{m_{t} a^{z} x_{t}}{a t^{z}}=F-k_{t} x_{t}-\frac{R_{t} a x_{t}}{a t}+A_{t} \Delta P
$$

, donde $m_{t}$ es la masa del pistón (placa), $F$ la fuerza aplicada sobre éste por el impulso dado por la cuerda a través de la vía mecánica del puente; $A_{t}$ el área del pistón y $R_{t}$ la resistencia al movimiento. El término $A_{t} \Delta P$, representa el cambio de presión por unidad de área del sistema frente a la excitación, en este caso de la placa armónica. (Para mayores detalles en el desarrollo del modelo de osciladores en el cuerpo de la guitarra, se recomienda revisar el trabajo de Wright [1]).

Para efectos del análisis visual y cuantitativo, se puede relacionar cada componente de esta ecuación con las variables físico-constructivas que influyen en la calibración sonora de una placa armónica. La elección de la madera determina la resistencia al movimiento que la placa tendrá, de acuerdo a las constantes de elasticidad (módulos de Young y Cizalle, principalmente), lo que con su elección en valores adecuados en definitiva otorga mayor permeabilidad al estímulo vibratorio, logrando una placa resonante "viva". Este breve análisis físico es llevado a cabo por un luthier mediante la ya mencionada técnica de golpeteo y de reconocimiento de puntos de alta vibración en la pieza de madera. A su vez, la masa total del pistón superior vibrante se determina también por las condiciones físicas propias del material utilizado en la placa, así como por la adhesión posterior del sistema de barraje en la calibración.

El estudio aislado de esta componente facilita el análisis en la adhesión de un sistema de barraje o bien de cualquier cambio físico que se realice sobre la placa armónica. Esta elección en las condiciones de trabajo se justifica pues la calibración previa en condición libre es una técnica tradicional utilizada por luthiers y recomendada con el objeto de efectuar cambios que provoquen una caracterización previa única para la placa y posteriormente para el instrumento.

En relación a esta calibración previa, se sustenta el estudio de afinación de placas libres de Carruth [3], donde se señalan modos vibratorios libres que se generan para determinadas frecuen- 
cias, los cuales se aprecian en la figura 1.
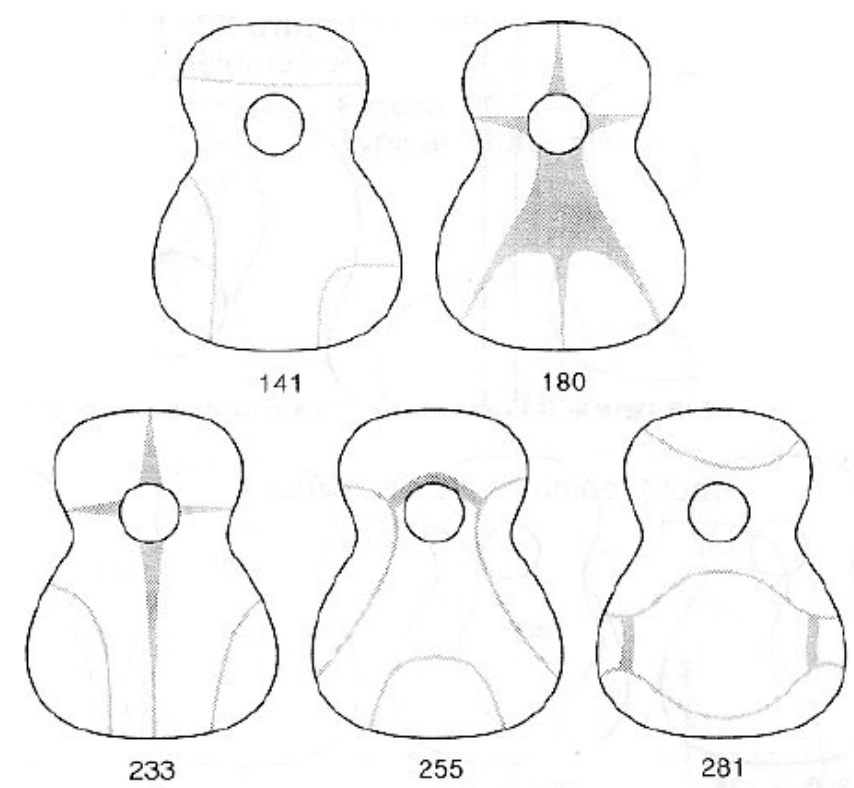

Figura 1: Modos de vibración en placa armónica en condiciones libres [3].

\section{TRABAJO EXPERIMENTAL}

\subsection{MATERIALES}

El trabajo experimental se realizó en dos placas armónicas de distinta especie: una construida a través de la manufactura especializada del luthier Nicanor Oporto Martínez, y otra desacoplada de una guitarra de construcción en serie marca Santana modelo CB390. En un primer caso se trabajó
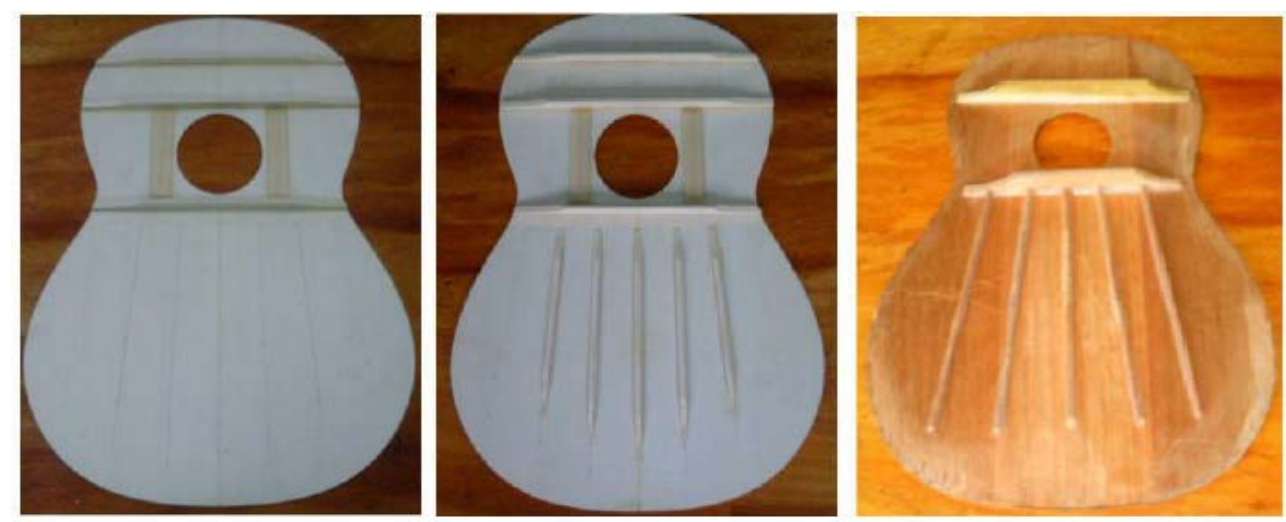

Figura 2: Placas armónicas utilizadas en trabajo experimental: placa Oporto sin barraje (I), placa Oporto con sistema de barraje Torres (II) y placa Santana (III).

con la placa Oporto sin sistema de barraje, para luego adherir un sistema de barraje tradicional 
Torres en el segundo caso. Finalmente, se compararon los resultados visuales y cuantitativos con la placa Santana. En la figura 2 se pueden apreciar las tres placas utilizadas en este trabajo.

\subsection{MONTAJE Y PROCEDIMIENTO}

Se realiza el montaje de la placa armónica sin puente con un excitador mecánico (shaker) Brüel \& Kjaer, situado en el eje central de la posición tentativa del puente (tomando como referencia la posición del puente desacoplado de la placa Santana), punto que se asumió como origen para la ubicación de futuros puntos de medición. Una vista general del montaje y punto de excitación se aprecia en la figura 3.

Se excita con un tono puro de amplitud media de 0.47 (VAC), escogida luego de pruebas previas para la generación de patrones nodales, en las que se requirió también del uso de soportes. El diseño de éstos se pensó con el objeto de generar un punto de mínimo contacto que no generase mayor perturbación en la vibración natural de la placa. Se realizaron pruebas en las que se buscaron zonas de mínima vibración, criterio con el que se determinaron dos puntos simétricos situados en las cercanías de la barra transversal inferior del barraje aplicado en el lóbulo superior, como se ve en la figura 3.
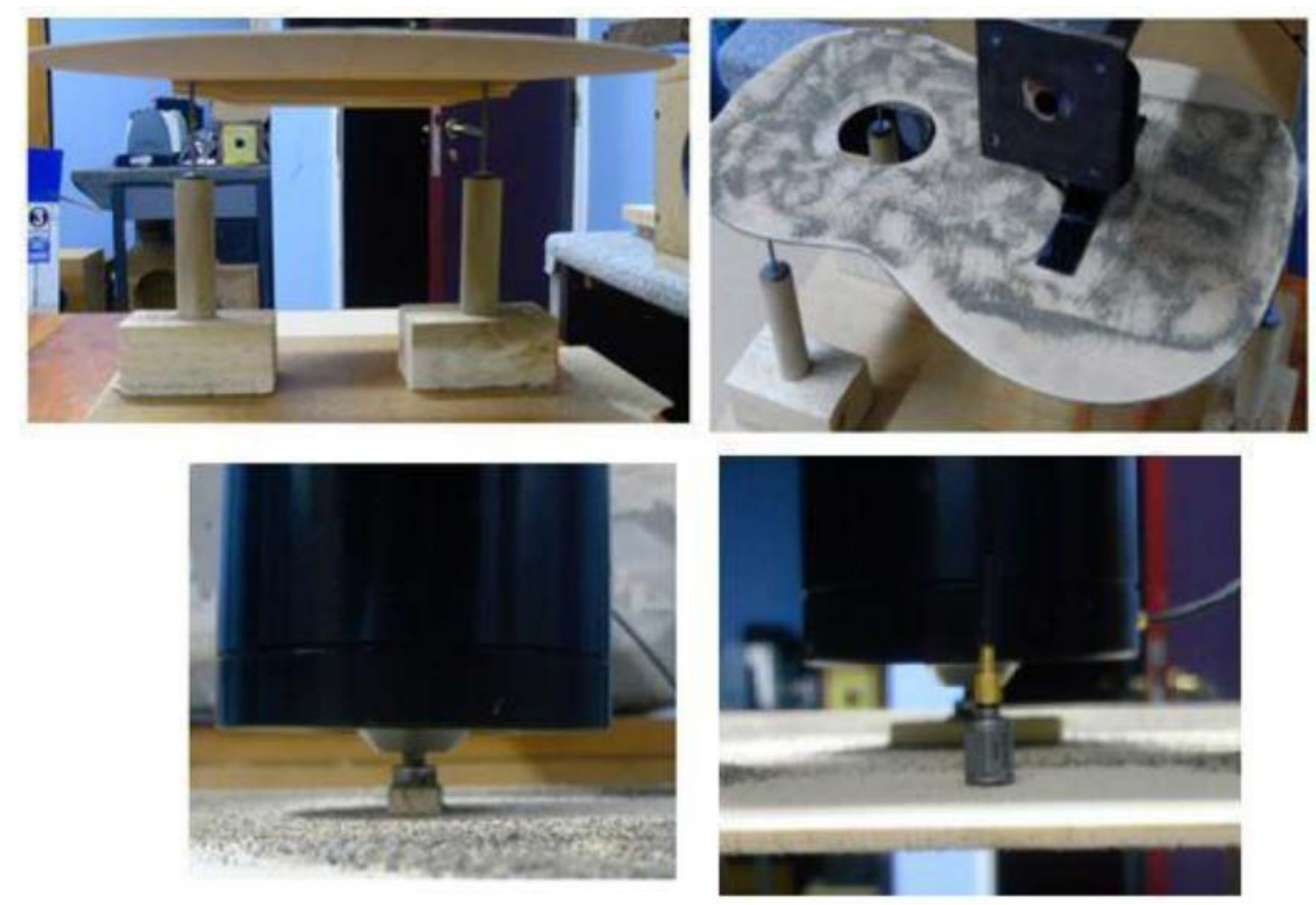

Figura 3: Vistas del montaje, punto de excitación y de medición con acelerómetro.

Para generar modos, el procedimiento consiste en distribuir de manera uniforme el material granulado (arena fina de mar en este caso), para luego realizar un barrido de frecuencias en el que 
se localizan fácilmente las resonancias por el movimiento de la arena. Luego se redistribuye la arena para generar un modo distinto. Para el proceso de medición, captura y análisis de datos, se trabajó con la interfaz Symphonie en conjunto con el software dBFA 4.1, donde se calibra para la medición de aceleración con el acelerómetro Brüel \& Kjaer serie 51145. Éste se sitúa en puntos de máxima vibración (antinodos), para cada patrón nodal ya identificado en el proceso anterior. Se debe señalar que el peso del acelerómetro es de 1.45 gramos, que a pesar de ser liviano, influye de manera notoria en la distribución de líneas nodales. Por esta razón se establecieron tres intervalos de medición consecutivos de 10 segundos, para la captura de datos, con el objeto de aproximar la vibración real del modo libre. Así, mediante el software se aplica un proceso de transformada rápida de Fourier (FFT) para los datos en captura, que permite analizar gráficamente la respuesta armónica en frecuencia de la placa frente a la señal de excitación, registrándose la amplitud del nivel de aceleración $(\mathrm{dB})$, para cada gráfica.

\section{RESULTADOS Y DISCUSIÓN}

\subsection{VISUALIZACIÓN DE MODOS}

En la figura 4 se muestran las principales resonancias halladas en el proceso de identificación de modos para las tres placas. Se revisan aquí sólo los modos generados que presentan similitud ya sea en frecuencia o bien en la forma de su patrón nodal para los tres casos: existe un número mayor de resonancias y patrones nodales que son propios para cada caso, así como una serie de modos débiles (o de poca definición) que se generan en torno de las frecuencias de resonancia de modos fuertes.

Se encontraron cuatro resonancias que coinciden para los tres casos y que además presentan un nivel de aceleración considerable en los $201 \mathrm{~Hz}, 290 \mathrm{~Hz}, 380 \mathrm{~Hz}$ y $473 \mathrm{~Hz}$. En general y para el caso de la placa Oporto sin barraje, se tiene un comportamiento vibratorio libre en el lóbulo inferior, y en todo el rango de frecuencias de trabajo de la guitarra, pero con mayor notoriedad en frecuencias superiores a los $200 \mathrm{~Hz}$. Se aprecia una situación similar en el segundo caso cuando se adhiere el barraje, pero con un esperado efecto de traslación de frecuencias para patrones de líneas nodales similares para frecuencias bajas principalmente (inferiores a $200 \mathrm{~Hz}$ ), con una traslación que puede variar entre los 20 hasta los $50 \mathrm{~Hz}$ hacia frecuencias superiores e incluso inferiores, en algunos casos.

Este efecto se resalta en la placa Santana donde la adhesión del barraje presenta mayor elasticidad, como también la madera posee poca permeabilidad a la transmisión de la señal, lo que provoca un cambio en los patrones nodales generales observados en el caso anterior. Aún así, se tienen algunas frecuencias que coinciden generando resonancias de consideración, como en los 201 $\mathrm{Hz}$, donde se aprecia un patrón nodal similar entre el caso sin barras y el segundo caso con sistema 

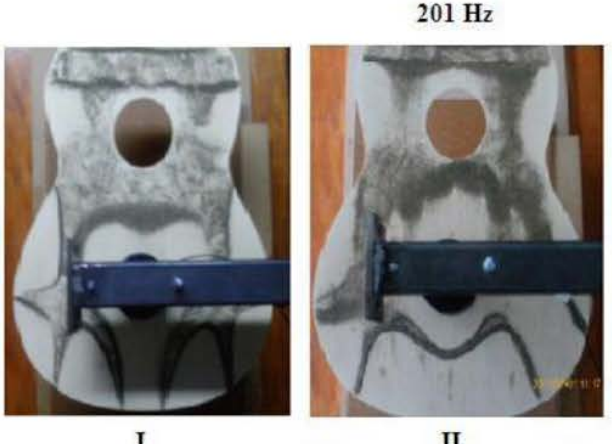

II

$290 \mathrm{~Hz}$

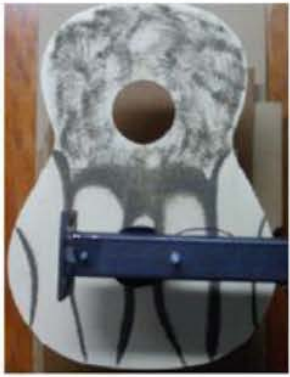

I

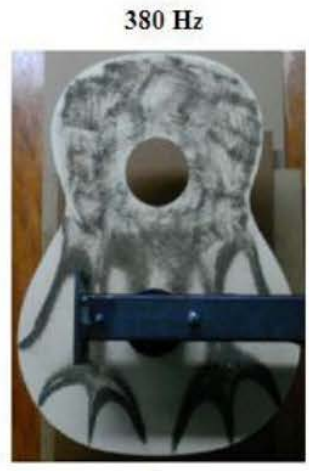

I

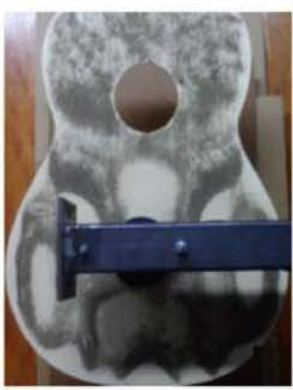

I

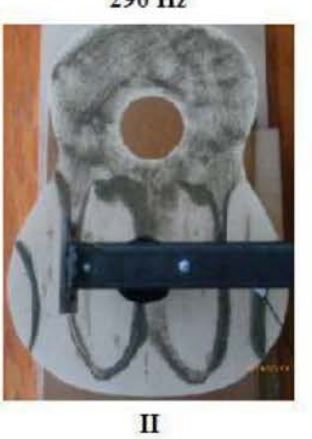

$415 \mathrm{~Hz}$

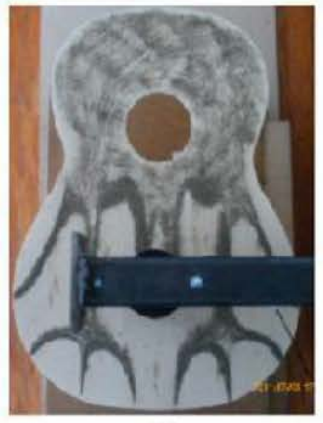

II

$473 \mathrm{~Hz}$

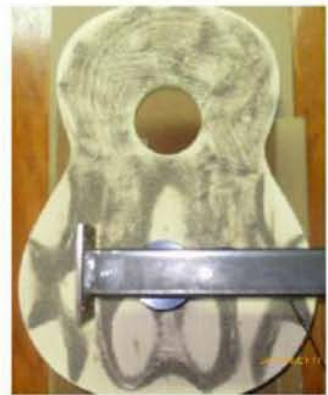

II

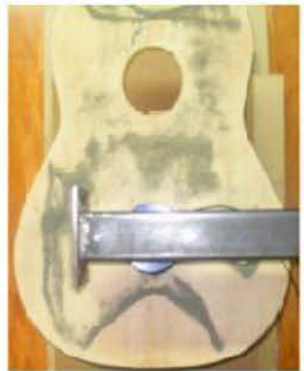

III

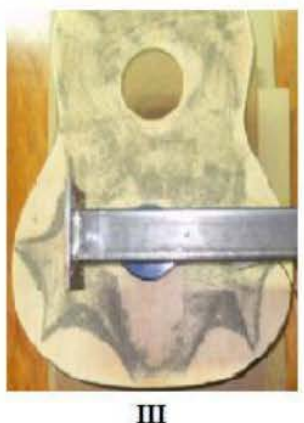

$380 \mathrm{~Hz}$
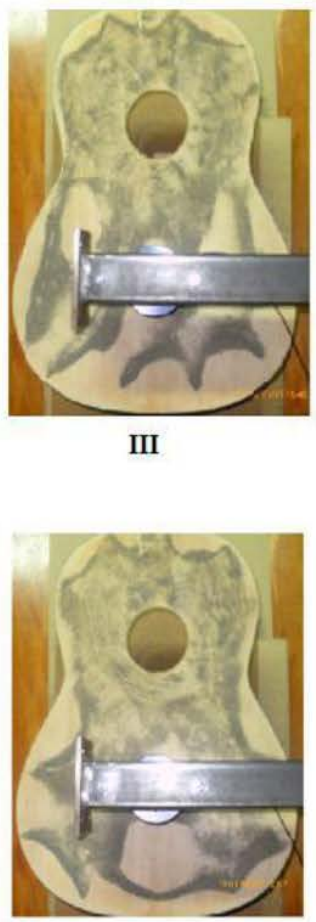

III

Figura 4: Patrones de líneas nodales hallados en la placa Oporto sin barras (I), placa Oporto con barraje (II) y placa Santana (III). 
de barraje adherido en la placa Oporto. En la placa Santana, sin embargo, se tiene un resultado poco claro y que no presenta ningún orden en el patrón nodal.

Siempre es posible realizar una comparación válida entre los casos II y III, ya que se tienen las mismas condiciones estructurales (no así físicas). De acuerdo a esto, se aprecia que en la siguiente resonancia en $290 \mathrm{~Hz}$, se da una semejanza notoria en los casos I y II con leves diferencias provocadas por el aumento de masa en el caso II. En el caso III, se obtiene un patrón totalmente distinto para la misma frecuencia.

Las siguientes resonancias en $380 \mathrm{~Hz}$ para la placa Oporto sin barras (I) y la placa Santana (III) y en $415 \mathrm{~Hz}$ para la placa Oporto con sistema de barraje (II), se encuentran en la zona de frecuencias de afinación tentativas para una placa armónica: donde pueden darse las mayores amplitudes en la vibración, que se reafirman en la prueba del golpe realizada por el luthier, donde se encuentra el punto dulce de excitación (llamado así por luthiers), en el cual la placa tiene una resonancia notoria, natural y de gran amplitud. En la escala musical estas frecuencias son cercanas al F\#4 en 369 Hz y al G4 en 392 Hz. Se observa en los resultados obtenidos un patrón común en los tres casos, el que nuevamente tiene mayor definición en la placa Oporto para los casos I y II, entre los que se comprueba el efecto de traslación de la frecuencia desde $380 \mathrm{~Hz}$ a $415 \mathrm{~Hz}$ que corresponde a la frecuencia de G\#4. En la placa Santana, se tiene una tendencia a cumplir el mismo patrón, pero con poca definición y claridad.

A partir de los $450 \mathrm{~Hz}$ en adelante, los máximos empiezan a ser cada vez más numerosos y cercanos entre sí formando patrones similares a los hallados en los $473 \mathrm{~Hz}$ para los tres casos. Se observan algunas modificaciones entre los patrones de los casos I y II, pero distinto para el caso III nuevamente.

\subsection{ANÁLISIS CUANTITATIVO}

Se muestran las FFT obtenidas para los modos vibratorios correspondientes a las frecuencias de $201 \mathrm{~Hz}$ y de $380 \mathrm{~Hz}$ (ó 415 en el caso II) analizados en la sección anterior, en las figuras 5 y 6. Se indican sólo estas gráficas pues en ellas es posible resumir los efectos cuantitativos observados, de acuerdo a las diferencias ya mencionadas entre casos. En la figura 5 se tienen las FFT de la resonancia hallada en los $201 \mathrm{~Hz}$ para los tres casos. Para los casos I y II se tiene un ordenamiento y decaimiento armónico suave y similar, que en términos de amplitud (nivel de aceleración en $\mathrm{dB}$ ) se reafirma obteniéndose $155 \mathrm{~dB}$ para el caso I en la fundamental y de $153 \mathrm{~dB}$ en el caso II, en el mismo punto de medición $(8,-10)$, en el extremo inferior derecho de la placa. Si bien existe una diferencia, esta no es notoria a grandes rasgos. Un resultado particular se tiene en la gráfica de la placa Santana. En ella se obtiene un máximo de amplitud en el tercer armónico en 
$607 \mathrm{~Hz}$, registrándose $147 \mathrm{~dB}$, superando a la fundamental en $201 \mathrm{~Hz}$ que registra $143 \mathrm{~dB}$. Esto demuestra la falta de condiciones naturales, físicas y elásticas de esta placa para la transmisión 'limpia' de una señal determinada, lo que en definitiva influye negativamente en las posibilidades de calibración tonal de ella: se tendría aquí una resonancia totalmente incongruente con la frecuencia de excitación.

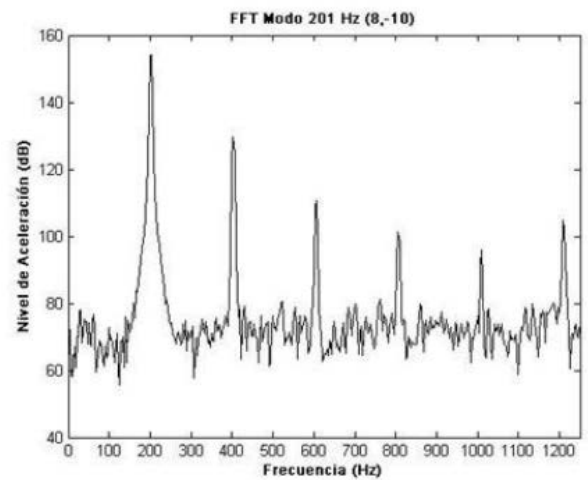

I

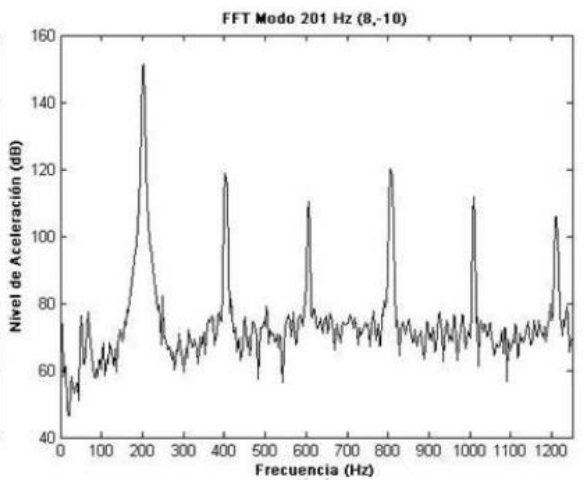

II

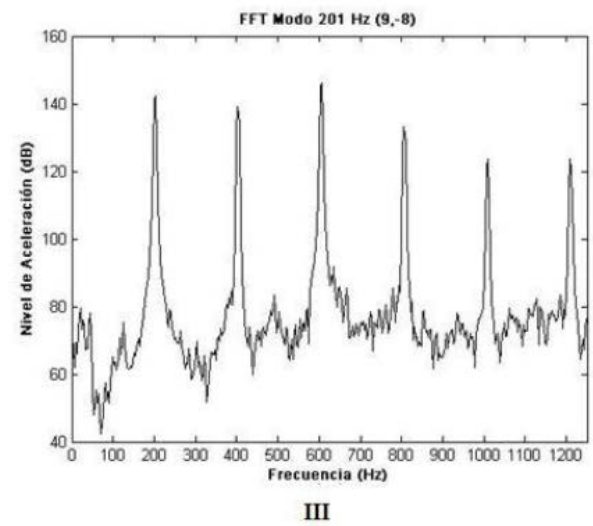

Figura 5: FFT's obtenidas en el modo vibratorio de $201 \mathrm{~Hz}$ para los casos de la placa Oporto sin barraje (I), placa Oporto con barraje (II) y placa Santana (III).

En la figura 6 se muestran la FFT's obtenidas para las resonancias en $380 \mathrm{~Hz}$ para el caso I y III y de $415 \mathrm{~Hz}$ en el caso II. En la primera gráfica correspondiente al caso I, se tiene un decaimiento ordenado en las amplitudes de los tres primeros armónicos registrándose un nivel de $154 \mathrm{~dB}$ en la fundamental para el punto $(-11,0)$, en el extremo izquierdo del lóbulo inferior. Para la frecuencia de $415 \mathrm{~Hz}$ en el caso II, que presentó un patrón nodal exactamente igual al caso I en $380 \mathrm{~Hz}$, se tiene una situación similar en la gráfica donde se presenta un patrón de decaimiento ordenado, inclusive en los armónicos de alto orden. Para esta resonancia se registra una amplitud de $153 \mathrm{~dB}$ en el punto simétrico contrario al de medición en el caso I, en $(11,0)$ y de $149 \mathrm{~dB}$ en $(-11,0)$. Este efecto se repite en el primer caso pero en una situación geométrica inversa, donde se obtiene un peak mayor en el punto (-11,0) como ya se mencionó. Se ratifica así la similitud en la amplitud entre los casos I y II. Finalmente para el caso III, se observa un comportamiento similar al de los casos I y II, donde 

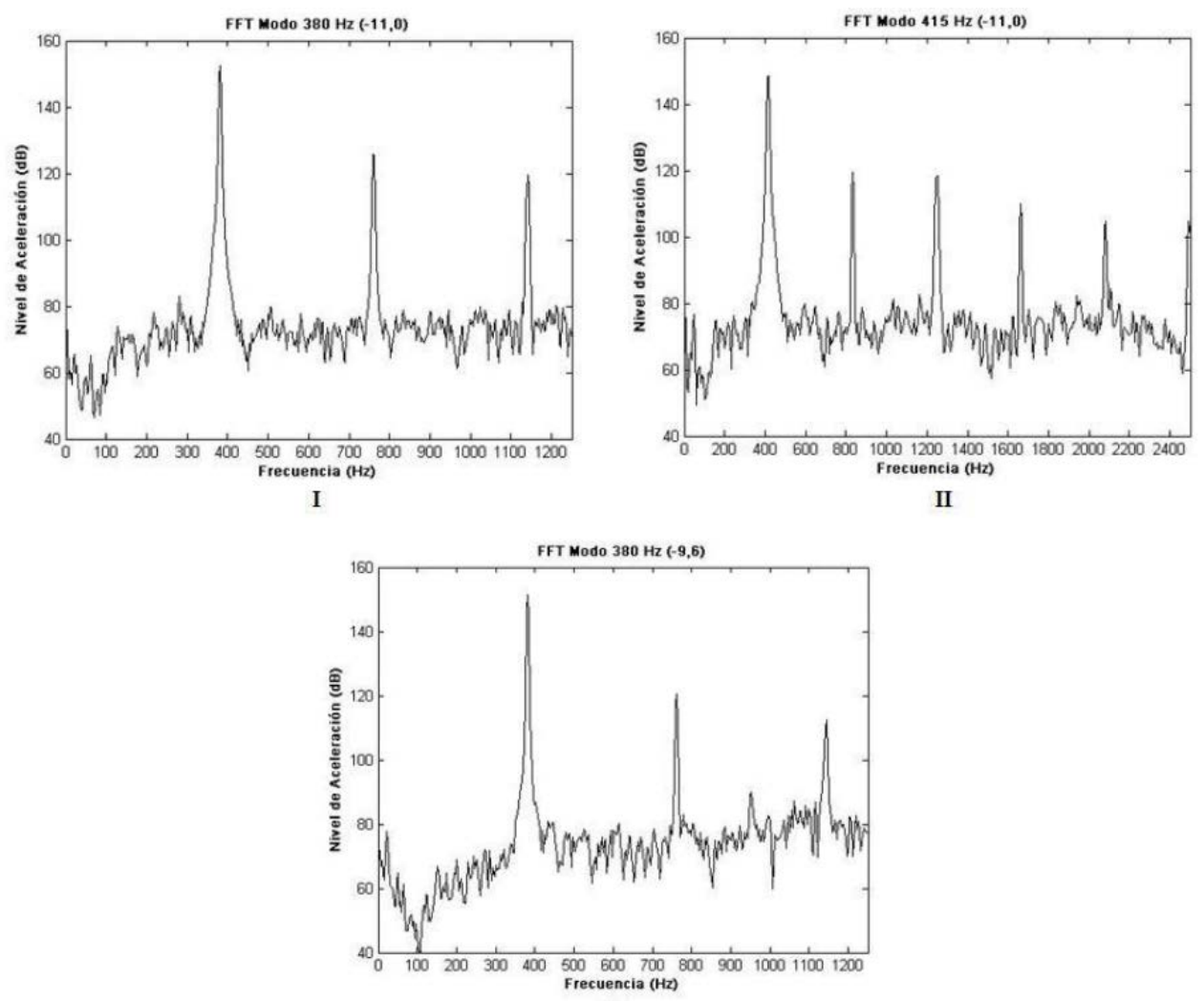

III

Figura 6: FFT's obtenidas en el modo vibratorio de $380 \mathrm{~Hz}$ para los casos de la placa Oporto sin barraje (I), y placa Santana (III). FFT en $415 \mathrm{~Hz}$ para la placa Oporto con barraje (II).

se registra una amplitud de $151 \mathrm{~dB}$ en la fundamental. Se comprueba así la tendencia que muestra la placa Santana en formar un patrón de líneas nodales similar al de los casos anteriores, pero con una menor amplitud en esta resonancia.

\section{CONCLUSIONES}

En términos generales se comprueba la eficiencia de la placa Oporto en cuanto a la transmisión de la señal vibratoria de una manera uniforme y simétrica en el lóbulo inferior de la placa. El principal efecto que muestra la adhesión de barraje es la traslación de patrones nodales en frecuencias inferiores a $200 \mathrm{~Hz}$, pero que mantiene y resalta amplitudes en modos con formas similares (o iguales), modificando también la ubicación de la frecuencia de resonancia de la placa, como se dio en $415 \mathrm{~Hz}$ del caso II, donde se invierte el patrón geométrico de amplitud con su modo semejante en $380 \mathrm{~Hz}$ del caso I.

La placa Oporto demuestra además similitud con los patrones hallados por Carruth [3], en frecuencias altas y bajas, a pesar de las diferencias en las condiciones experimentales de ambos 
trabajos.

La placa Santana en contraste demuestra su poca efectividad en la transmisión de una señal, como ocurre en la frecuencia de $201 \mathrm{~Hz}$, y en general en el poco orden en el decaimiento y orden de los armónicos observado en gráficas de diversos modos excitados. Estos efectos son atribuibles a la mala calidad de la madera y a sus condiciones elásticas. Por otra parte el sistema de barraje adherido con una distribución asimétrica y con un pegamento sintético y elástico a la vez, puede contribuir en la deficiente respuesta de la placa para la formación de patrones nodales desordenados y poco definidos, como también en las gráficas obtenidas.

\section{REFERENCIAS}

[1] WRIGHT, Howard, "The Acoustics and Psychoacoustics of the guitar". Tesis para el grado de Doctor en Filosofía, Departamento de física y astronomía, Universidad de Gales. Cardiff, 1996.

[2] CHRISTENSEN O., "An oscillator model for analysis of guitar sound pressure response", Magazine Acustica 1984. EUA.

[3] CARRUTH Alan, "Free plate tuning, part three: Guitars". Revista American Lutherie $\mathrm{N}^{\circ} 30$, 1992. EUA. 